\title{
Análisis preliminar del efecto de la edad y la escolaridad en adultos sanos en el rendimiento del Sydney Language Battery (Sydbat) en español de Chile
}

\author{
Camila Foncea-González ${ }^{\text {a, } \boldsymbol{~}}$, Teresa Julio-Ramos ${ }^{\text {a, \& }}$, Gada Musa-Salech ${ }^{\text {b }}$, Patricia Lillo-Zurita ${ }^{\text {c, d }}$, María \\ Elena León-Bustos ${ }^{\text {a }}$, Valentina Muñoz-Oyarzún ${ }^{\text {a }}$, Paula Soto-Valdés a y Carolina Méndez-Orellana ${ }^{\text {a, * }}$ \\ ${ }^{a}$ Carrera de Fonoaudiología, Departamento de Ciencias de la Salud, Pontificia Universidad Católica de Chile, Chile \\ ${ }^{\mathrm{b}}$ Clínica Universidad de Los Andes, Chile \\ ${ }^{\mathrm{c}}$ Departamento de Neurología Sur, Universidad de Chile, Chile \\ ${ }^{\mathrm{d}}$ Gerosciences Center for Brain Health and Metabolism (GERO), Chile \\ ${ }^{\star}$ Contribución equitativa (Equally contribution)
}

\section{RESUMEN}

Existen 3 variantes de afasia progresiva primaria (APP), que se distinguen según el dominio lingüístico predominantemente alterado: no fluente, logopénica y semántica. Una herramienta que ayuda a su clasificación es el Sydney Language Battery (Sydbat), el cual no se encuentra validado en Chile. El objetivo del presente trabajo es analizar la posible relación entre edad, años de escolaridad y rendimiento obtenido en las subpruebas del Sydbat en adultos sanos. Participaron 38 adultos sanos a los que se les aplicó el test. El promedio de edad fue 56,8 años ( $\mathrm{DE}=8,3)$ y tenían una media de 13,8 años de escolaridad $(\mathrm{DE}=$ 3,9). Todos provenían de la Región Metropolitana. Se realizaron correlaciones de Pearson y Spearman, para explorar la correlación entre el rendimiento en las subpruebas, la edad y la escolaridad. Considerando la variabilidad en el rendimiento en las subpruebas, se realizaron modelos uni y multivariados. Se encontraron correlaciones significativas entre edad y la subprueba comprensión $(\mathrm{p}<0,05)$ y los años de escolaridad con las subpruebas asociación semántica $(\mathrm{p}<0,001)$ y nominación $(\mathrm{p}<0,01)$. En personas con más de 12 años de escolaridad, el puntaje en la subprueba asociación semántica varió aproximadamente 2 puntos, con respecto a personas con menos de 12 años de escolaridad ( $<<0,01)$. Se concluye que las variaciones en el rendimiento del test, dependen la edad y la escolaridad. A mayor edad, menor es el rendimiento en la subprueba de comprensión. A mayor escolaridad, mayor es el rendimiento en las subpruebas de asociación semántica y nominación. El efecto mencionado debe ser considerado en el proceso de validación de Sydbat.

\section{Preliminary analysis of the effect of age and education in healthy adults on the performance of the Sydney Language Battery (Sydbat) in Chilean Spanish}

\begin{abstract}
There are 3 variants of primary progressive aphasia (APP), which are distinguished according to the predominantly altered linguistic domain: non-fluent, logopenic, and semantic. A tool that helps in its classification is the Sydney Language Battery (Sydbat), which is not validated in Chile. The objective of this work is to analyze the possible relationship between age, years of schooling and performance obtained in the Sydbat subtests in healthy adults. 38 healthy adults participated in the test. The average age was 56.8 years $(\mathrm{SD}=8.3)$ and they had a mean of 13.8 years of schooling $(\mathrm{SD}=3.9)$. They all came from the Metropolitan Region. Pearson and Spearman correlations were performed to explore the correlation between subtest performance, age, and schooling. Considering the variability in performance in the subtests, univariate and multivariate models were performed. Significant correlations were found between age and the comprehension subtest $(\mathrm{p}<0.05)$ and the years of schooling with the semantic association $(\mathrm{p}<0.001)$ and nomination $(\mathrm{p}<0.01)$ subtests. In participants with more than 12 years of schooling, the score in the semantic association subtest varied approximately 2 points, with respect to people with less than 12 years of schooling $(\mathrm{p}<0.01)$. It is concluded that the variations in test performance depend on age and education. The older participants are, the lower their performance on the comprehension subtest. The higher the schooling, the higher the performance on the semantic association and nomination subtests. The mentioned effect must be considered in the Sydbat validation process.
\end{abstract}

*Autor/a correspondiente: Carolina Méndez-Orellana

Recibido: 25-08-2020

Email: carolinamendez@uc.cl

Palabras clave:

Afasia Progresiva Primaria; Sydbat; demencia; envejecimiento normal; nivel educacional
Keywords: Primary Progressive Aphasia; Sydbat; dementia; aging; educational attainment 


\section{INTRODUCCIÓN}

La afasia progresiva primaria (APP) es un cuadro heterogéneo que se caracteriza por una atrofia cerebral en la zona perisilviana, manifestándose en distintos trastornos neurodegenerativos, tales como en la demencia Frontotemporal o la demencia de tipo Alzheimer (Baeza et al., 2012; Gorno-Tempini et al., 2011; Matías-Guiu \& García-Ramos, 2013; Serrano et al., 2005). El diagnóstico de la APP se centra en las alteraciones observadas predominantemente en el dominio del lenguaje sin la afectación de otras funciones cognitivas. Generalmente el cuadro de APP se puede manifestar entre los 40 y 70 años, motivo por el cual es descrita como un tipo de demencia de inicio temprano (Baeza et al., 2012). Se describen 3 variantes de APP: una variante no fluente, una variante semántica y una variante logopénica (GornoTempini et al., 2011). Estas 3 variantes presentan distinto nivel de severidad en las alteraciones a nivel de comprensión y expresión del lenguaje, así como también daño específico a un nivel del lenguaje (nivel fonológico, semántico o nivel sintáctico) (Custodio et al., 2012; Gorno-Tempini et al., 2011; Matías-Guiu \& García-Ramos, 2013; Savage et al., 2013). La variante no fluente se caracteriza por alteraciones a nivel de procesamiento gramatical que eventualmente puede ir acompañado con la presencia de apraxia del habla. En la variante semántica, los signos más característicos son la anomia severa y la alteración en la comprensión de palabras aisladas, en especial de aquellas de baja frecuencia. A diferencia de la variante no fluente, en la variante semántica el lenguaje es fluente y adecuado gramaticalmente. Por último, la variante logopénica se caracteriza por la presencia de parafasias fonológicas tanto en tareas de nominación como en el habla espontánea y alteraciones en la repetición de oraciones, sin embargo no se observan alteraciones gramaticales o problemas articulatorios.

Dada la diversidad de alteraciones lingüísticas en las variantes de APP, el diagnóstico de estos subtipos es un desafío, debido a la falta de instrumentos de evaluación que faciliten la detección de alteraciones lingüísticas específicas (Sapolsky et al., 2014; Savage et al., 2013). Actualmente, no existen herramientas validadas en español para la evaluación lingüística específica del cuadro de APP. La utilización de instrumentos específicos es fundamental para una clara y precisa caracterización de este tipo de afasia (Gorno-Tempini et al., 2011; Knopman et al., 2007; Sapolsky et al., 2010). Comúnmente la caracterización de las alteraciones que presentan los pacientes con APP se realiza principalmente con 2 tests de cribado cognitivo, el Montreal Cognitive Assessment (MoCA) (Delgado et al., 2019) y el Minimental State Examination (MMSE) (González-Hernández et al., 2009). Ambas herramientas han sido diseñadas para estudiar cuadros de demencia y deterioro cognitivo, por lo que no están destinadas al estudio de las alteraciones lingüísticas (Delgado \& Salinas, 2009). Además, la literatura reporta que los puntajes de corte en este tipo de instrumentos son afectados por variables demográficas tales como los años de escolaridad y la edad (González-Hernández et al., 2009).

La escolaridad es una variable que no debe ser ignorada al momento de validar una herramienta, puesto que está estrechamente relacionada con el desempeño de habilidades lingüísticas como la nominación, comprensión oral y comprensión gramatical (Burke \& Shafto, 2004; Véliz et al., 2010). Lo anterior toma relevancia dado que en la población chilena el promedio de escolaridad (12 años) disminuye en adultos mayores (Ministerio de Desarrollo Social \& Subsecretaría de Evaluación Social, 2015). También, la variable edad es relevante en el rendimiento lingüístico, debido a las diferencias encontradas entre un adulto joven y uno mayor (López Higes et al., 2013; Soares \& Ortiz, 2009).

En 2013 se creó en Australia el "Sydney Language Battery" (Sydbat) con el objetivo de caracterizar las variantes de APP mediante 4 subpruebas: nominación, asociación semántica, comprensión oral y repetición (Savage et al., 2013). Esta herramienta diagnóstica demostró tener una buena validez convergente con coeficientes de correlación de Pearson dentro de un rango de $\mathrm{r}=0,67$ para repetición y $\mathrm{r}=0,95$ para nominación. Adicionalmente, este test demostró tener una buena fiabilidad interevaluador, ser válida y confiable para determinar las variantes de APP en pacientes de habla inglesa (Savage et al., 2013).

Por otra parte, este test permite a los profesionales implicados en el diagnóstico y tratamiento de la APP, implementar una batería única para caracterizar la sintomatología clínica lingüística del paciente con APP, evitando así extraer subtest de otras pruebas diagnósticas que no están diseñadas para este tipo de pacientes.

En Chile, la población de adultos mayores tiene un alto riesgo de sufrir enfermedades neurodegenerativas (Gajardo \& Monsalves, 2013). Es necesario, por tanto, contar con herramientas validadas en el contexto nacional que faciliten el diagnóstico temprano de la presencia de cuadros de demencia, entre ellos, las variantes del cuadro APP. La validación chilena del Sydbat permitirá brindar a las personas con APP una terapia oportuna y dirigida a sus necesidades cognitivas y comunicativas específicas (Sapolsky et al., 2014).

Debido a los planteamientos previo, el objetivo de este estudio es analizar la posible relación entre edad, años de escolaridad y el 
rendimiento obtenido en las subpruebas de la versión chilena del Sydbat, en adultos sanos mayores de 40 años de la Región Metropolitana.

\section{METODOLOGÍA}

El estudio que se presenta es un piloto que tiene un diseño de tipo observacional-analítico de corte transversal. La muestra fue seleccionada por conveniencia, invitando a participar a adultos chilenos sanos mayores de 40 años, residentes en la Región Metropolitana de Chile. Los criterios de inclusión fueron: a) ser hablante nativo de español, b) tener más de 40 años, y c) residir en la Región Metropolitana. Los criterios de exclusión fueron: a) poseer alguna discapacidad auditiva y/o visual severa, b) tener diagnóstico de dislexia, c) tener de enfermedad psiquiátrica o neurodegenerativa, d) poseer antecedentes de enfermedades neurológicas y e). obtener un puntaje inferior a 26 en el Montreal Cognitive Assessment (MoCA), lo cual sugiere la presencia de deterioro cognitivo (Delgado et al., 2019).

\section{Participantes}

Se efectuó un pilotaje con 10 voluntarios sanos para la adaptación lingüística y cultural de la batería Sydbat en Chile. A partir de este pilotaje, se calculó el tamaño muestral esperado para el proceso de validación que corresponde a 62 participantes. Sin embargo, no se pudo completar el tamaño muestral, debido a los sucesos sociales ocurridos el segundo semestre del año 2019 en Chile. El año 2020 no se pudo retomar la actividad debido a la pandemia del COVID-19. A pesar de las dificultades mencionadas, se logró que 51 personas participaran en el estudio, de los cuales 13 no cumplieron con el puntaje de corte en el test MoCA. Por lo tanto, la muestra final se constituyó de 38 participantes. Sus características se presentan en la Tabla 1.

Todos los participantes firmaron un consentimiento informado previo a la evaluación. Además, el estudio fue aprobado por el Comité Ético Científico de Ciencias de la Salud de la Pontificia Universidad Católica de Chile (190510002).

\section{Características del Test Sydbat}

El test Sydbat evalúa habilidades lingüísticas a nivel de palabras aisladas y para ello cuenta con cuatro subpruebas: nominación, repetición, comprensión y asociación semántica. Cada subprueba se puntúa 0 y 1 punto y el puntaje máximo es de 30 puntos. Los estímulos se presentan en modalidad digital (tablet o computador) y se ordenan en base a la dificultad según parámetros lingüísticos, de imaginabilidad, frecuencia y complejidad silábica (Savage et al., 2013).

Tabla 1. Características demográficas de los participantes.

\begin{tabular}{ll}
\hline Variable & Voluntarios $(\mathrm{n}=38)$ \\
\hline Edad & \\
$\quad$ Años, media, (DE), [rango] & $56,8,(8.3)[40-77]$ \\
Género & $18(47,3)$ \\
$\quad$ Femenino, n (\%) & $20(52,6)$ \\
$\quad$ Masculino, n (\%) & \\
Lateralidad & $33(86,8)$ \\
$\quad$ Diestro, n (\%) & $3(7,8)$ \\
$\quad$ Zurdo, n (\%) & $2(5,2)$ \\
$\quad$ Ambidiestro & \\
Escolaridad & $13,8(3,9)[6-23]$ \\
$\quad$ Años, media, (DE), [rango] & $11(28,9)$ \\
$\quad<12$ años, n (\%) & $27(71)$ \\
$\quad \geq 12$ años, n (\%)
\end{tabular}

DE: desviación estándar.

\section{Adaptación chilena Test Sydbat}

La adaptación en español de Chile del test Sydbat fue realizado por un comité de expertos, compuesto por 2 fonoaudiólogas, 1 neuropsicóloga y 2 neurólogas con más de 10 años de experiencia en la evaluación y tratamiento de pacientes con demencia. Se realizó acorde a las variables consideradas internacionalmente para la validación de instrumentos de evaluación en salud (Carvajal et al., 2011; Hilton \& Skrutkowski, 2002).

Las palabras de cada subtest se calibraron, según frecuencia, metría y composición silábica. La frecuencia de las palabras se determinó utilizando el diccionario de frecuencia de Sadowsky \& Martínez-Gamboa (2008). Por su parte, la metría y composición silábica, se estableció mediante el conteo de las sílabas y análisis de los fonemas que componen cada estímulo. Se realizaron 3 sesiones de evaluación del comité experto, tras las cuales se determinó la lista final de los estímulos y sus respectivas imágenes, eliminado contraste y fondos que pudieran generar confusión. La versión final obtuvo un índice de validez de contenido (IVC) de Lawshe de 0,92 entre los 5 jueces expertos. 


\section{Administración Test Sydbat}

El rendimiento lingüístico de los participantes fue evaluado a través de la Batería Sydbat. La primera subprueba es la de nominación que consiste en presentar una imagen a la vez. El participante debe responder mencionando lo que ve en la imagen. La omisión o cualquier error fonético, fonológico o semántico se considera incorrecto. Luego, se aplica la subprueba de repetición en la cual el participante debe repetir la palabra que el evaluador menciona sin apoyo visual del estímulo. Posteriormente, se aplica la subprueba de comprensión, en la que el evaluador presenta un estímulo auditivo y el participante debe seleccionar una imagen dentro de un grupo de estímulos visuales. El grupo de estímulos incluye la palabra objetivo y seis términos semánticamente relacionados. Finalmente, se aplica la subprueba de asociación semántica, donde el evaluador pronuncia una palabra y el participante debe seleccionar la imagen correspondiente dentro de los distractores.

\section{Análisis estadístico}

Para evaluar la distribución de las variables del estudio se aplicó la prueba de Shapiro-Wilk. En base a la distribución de normalidad de los datos, se realizaron correlaciones de Pearson y Spearman. Posteriormente, se efectuaron modelos lineales univariados, entre cada subprueba, edad y años de escolaridad. En base a la alta variabilidad de la variable comprensión, se realizó un modelo lineal multivariado con las variables edad, años de escolaridad y esta subprueba.

\section{RESULTADOS}

\section{Participantes}

En la Figura 1 se observan las puntuaciones de tres de las subpruebas del Test Sydbat. En el caso de las subpruebas asociación semántica y comprensión, la mediana se ubicó en el puntaje máximo (30 puntos). Existió mayor variabilidad en la subprueba de nominación, variando sus puntuaciones entre 25 y 30 puntos (Fig. 1). El puntaje en subprueba de repetición no fue considerado en el análisis puesto que presentó efecto techo.

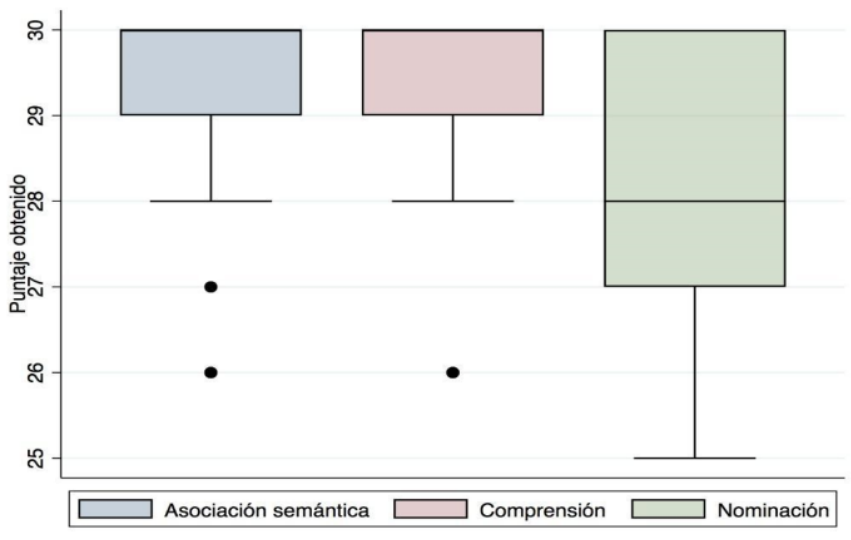

Figura 1. Puntuaciones de las subpruebas del test Sydbat en una muestra de adultos sanos de 40 años o más $(n=38)$.

La variable edad presentó sólo una correlación significativa (negativa) con el rendimiento en la subprueba de comprensión. A mayor edad, menor puntaje en esta subprueba. La variable escolaridad tuvo una correlación moderada con el rendimiento en la subprueba de asociación semántica y nominación. A mayor escolaridad, mayor es el puntaje obtenido en dichas subpruebas (Tabla 2).

Tabla 2. Matriz de correlaciones entre las subpruebas, edad, y años de escolaridad $(\mathrm{n}=38)$.

\begin{tabular}{lccc}
\hline & $\begin{array}{c}\text { Asociación } \\
\text { semántica }^{\text {a }}\end{array}$ & Nominación $^{\mathrm{b}}$ & Comprensión $^{\text {a }}$ \\
\hline $\begin{array}{l}\text { Edad } \\
\text { Años de } \\
\text { escolaridad }\end{array}$ & 0,01 & $-0,08$ & $-0,37^{*}$ \\
\hline
\end{tabular}

a Considerando que las subpruebas de asociación semántica y comprensión distribuyeron en forma sesgada (se constata mediante el test de Shapiro-Wilk) se obtuvo el coeficiente de correlación de Spearman.

${ }^{\text {b }}$ Se estimó el coeficiente de correlación de Pearson.

$* \mathrm{p}<0,05 ; * * \mathrm{p}<0,01 ; * * * \mathrm{p}<0,001$

En los modelos lineales univariados (Tabla 3), la edad demostró tener una relación significativa con la subprueba de comprensión, no así con las variables asociación semántica y nominación. Por cada año del participante, el puntaje disminuye en 0,04 puntos. La variable años de escolaridad demostró tener una relación significativa con las subpruebas de asociación semántica y 
nominación, debido a que sus puntajes varían en 2 y 0,2 puntos respectivamente, en personas con más de 12 años de escolaridad.

En cuanto al modelo lineal multivariado, se utilizó en la subprueba comprensión debido a su alta variabilidad. En este modelo, sólo la variable edad explicó cerca de un $18 \%$ en la variación de la puntuación en la subprueba de comprensión (Tabla $3)$.

Tabla 3. Modelos lineales univariados y multivariados para analizar la relación entre las subpruebas, edad, y años de escolaridad ( $\mathrm{n}=38)^{\text {a, }}$.

\begin{tabular}{lcccccc}
\hline & $\begin{array}{c}\text { Asociación } \\
\text { semántica }\end{array}$ & $\mathrm{R}^{2}$ & Nominación & $\mathrm{R}^{2}$ & Comprensión $^{\mathrm{d}}$ & $\mathrm{R}^{2}$ \\
\hline Modelos multivariados & & & & & & \\
Edad & 0,01 & $<1,0$ & $-0,02$ & $<1,0$ & $-0,04 * *$ & 9,2 \\
Años de escolaridad ${ }^{c}$ & $1,97^{* *}$ & 34,1 & $0,19^{* *}$ & 19,9 & 1,02 & 11 \\
Modelos multivariados $^{\text {Edad }}$ & - & - & - & - & $-0,04 * *$ & 17,8 \\
Años de escolaridad $^{c}$ & - & - & - & - & 0,91 & \\
\hline
\end{tabular}

${ }^{\mathrm{a}}$ Resultados expresados como coeficientes del modelo lineal (Beta).

b En el caso de los modelos lineales en que se tuvo como variable respuesta a las puntuaciones de las subpruebas asociación semántica y comprensión se utilizó un estimador de varianza robusta.

${ }^{\mathbf{c}}$ Resultados expresados para una cantidad mayor a 12 años de escolaridad.

${ }^{d}$ Se incluyó en el modelo multivariado a los años de escolaridad dado que su valor de significancia fue $\mathrm{p}<0,1$.

$* * \mathrm{p}<0,01$

\section{DISCUSIÓN}

El presente estudio analizó la relación entre las variables edad y años de escolaridad en el rendimiento en tres de las subpruebas del test Sydbat (versión en español para Chile) en personas sanas mayores de 40 años residentes de la Región Metropolitana. Los resultados muestran que la variable edad influye en el rendimiento en la subprueba de comprensión del test Sydbat, mientras que los años de educación influyen en el rendimiento de las subpruebas de asociación semántica y nominación.

Se encontró una relación significativa de carácter negativa entre las variables edad y comprensión lo que indica que, a mayor cantidad de años, menor es el puntaje obtenido en esta subprueba. Estos resultados están alineados con lo planteado por Saá (2006), la edad provoca un deterioro en sistemas centrales, como la memoria operativa, la cual tiene una repercusión en el sistema atencional, y en consecuencia conlleva a una afectación de la comprensión del lenguaje. Con respecto a las habilidades de asociación semántica y de nominación, se evidenció una correlación significativa positiva con respecto a los años de escolaridad, lo que señala que, a mayor cantidad de años de escolaridad, mayor es el puntaje obtenido. Diversos estudios (Lozano \& Ostrosky-Solís, 2006; Paolieri et al., 2018; Zanin et al., 2010) reportan el efecto de la escolaridad en el sistema léxico-semántico y describen que, a mayor escolaridad mejor es el rendimiento en pruebas que valoren dicho sistema. La razón de este efecto viene dada porque los años de escolaridad promueven habilidades metacognitivas y verbales que potencian el desarrollo del sistema léxico-semántico.

El trabajo expuesto presenta dos limitaciones que se comentan a continuación. Una refiere al reducido tamaño muestral, puesto que sólo se logró evaluar un $61 \%$ de la muestra propuesta para completar la validación de este instrumento. Ello restringió los análisis que se podían efectuar para estudiar la validación del test. La otra limitación se relacionó con la prueba de screening utilizada para controlar la presencia de deterioro cognitivo (MoCA). Esta prueba entrega un puntaje adicional a las personas con baja escolaridad. A pesar de ello, un $26 \%$ de la muestra inicial, que tenía una menor educación, no cumplió con su puntaje de corte. Esto repercutió en el estudio, porque redujo la variabilidad de años de escolaridad de la muestra, lo que implicó que la muestra se caracterizó por estar constituida por integrantes con un número relevante de años de educación. Por tanto, es necesario considerar este sesgo muestral para el proceso de validación del test Sydbat.

En futuros trabajos se debería cuidar este sesgo, por lo que es recomendable utilizar una escala clínica de demencia como la 
Clinical Dementia Rating de Hughes. La escala mencionada es un instrumento que considera la participación de personas con pocos años de escolaridad, ya que controla sus síntomas de deterioro cognitivo (Morris, 1997).

A pesar de las limitaciones mencionadas, los resultados obtenidos, aunque son preliminares, orientan el proceso de validación de la batería Sydbat. Al respecto, se destaca la necesidad de completar el tamaño muestral propuesto considerando la variabilidad de edad y nivel educacional. Además, se espera integrar voluntarios de otras regiones del país, para así obtener resultados más representativos de la población chilena.

\section{CONCLUSIÓN}

El test Sydbat es un instrumento de evaluación diseñado con la finalidad de clasificar los subtipos de APP. Los resultados preliminares de la versión en español de Chile de este test sugieren que el proceso de validación debe reconsiderar el test de MoCA para la selección de sus participantes, así como también controlar el efecto de las variables edad y años de escolaridad en las subpruebas de asociación semántica, nominación y comprensión.

\section{AGRADECIMIENTOS}

Las autoras agradecen a Eduardo Fuentes López por su apoyo en el análisis preliminar realizado para este estudio.

\section{REFERENCIAS}

Baeza, S., Bustos, C., \& Ovando, P. (2012). Logopenic Aphasia: A case report an review of the literature. Revista chilena de neuro-psiquiatría, 50(3), 166-173. https://doi.org/10.4067/S0717-92272012000300006

Burke, D., \& Shafto, M. (2004). Aging and Language Production. Current Directions in Psychological Science, 13(1), 21-24. https://doi.org/10.1111/j.09637214.2004.01301006.x

Carvajal, A., Centeno, C., Watson, R., Martínez, M., \& Rubiales, A. S. (2011). How is an instrument for measuring health to be validated? Anales Del Sistema Sanitario De Navarra, 34(1), 63-72. https://doi.org/10.4321/s113766272011000100007

Custodio, N., Castro, S., Herrera-Pérez, E., Lira, D., Prado, L. N. del, Cortijo, P., \& Montesinos, R. (2012). Afasias progresivas primarias: Las afasias de lenta evolución a demencia. Revista de Neuro-Psiquiatria, 75(3), 93-100. https://doi.org/10.20453/rnp.v75i3.1227

Delgado, C., Araneda, A., \& Behrens, M. I. (2019). Validación del instrumento Montreal Cognitive Assessment en español en adultos mayores de 60 años.
Neurología, 34(6), 376-385. https://doi.org/10.1016/j.nrl.2017.01.013

Delgado, C., \& Salinas, P. (2009). Evaluación de las alteraciones cognitivas en adultos mayores. Revista Hospital Clínico Universidad de Chile, 20, 244-251. https://www.redclinica.cl/Portals/0/Users/014/14/14/Publicaciones/Revista/altera ciones_adultos_mayores.pdf

Gajardo, J., \& Monsalves, M. J. (2013). Demencia, un tema urgente para Chile. Revista Chilena de Salud Pública, 17(1), 22-25. https://doi.org/10.5354/07195281.2013.26633

González-Hernández, J., Aguilara, L., Oporto, S., Araneda, L., \& Vásqueza, M. (2009). Normalización del Mini-Mental State Examination según edad y educación, para la población de Santiago de Chile. Revista Memoriza.com, 3, 23 34. http://www.memoriza.com/documentos/revista/2009/minimental2009_3_2334.pdf

Gorno-Tempini, M. L., Hillis, A. E., Weintraub, S., Kertesz, A., Mendez, M., Cappa, S. F., Ogar, J. M., Rohrer, J. D., Black, S., Boeve, B. F., Manes, F., Dronkers, N. F., Vandenberghe, R., Rascovsky, K., Patterson, K., Miller, B. L., Knopman, D. S., Hodges, J. R., Mesulam, M. M., \& Grossman, M. (2011). Classification of primary progressive aphasia and its variants. Neurology, 76(11), 1006-1014. https://doi.org/10.1212/WNL.0b013e31821103e6

Hilton, A., \& Skrutkowski, M. (2002). Translating Instruments Into Other Languages: Development and Testing Processes. Cancer Nursing, 25(1), 1-7. https://journals.1ww.com/cancernursingonline/Abstract/2002/02000/Translating_ Instruments_Into_Other_Languages_.1.aspx

Knopman, D. S., Boeve, B. S., Caselli, R. J., Graff-Radford, N. R., Kramer, J. H., Mendez, M. F., \& Miller, B. L. (2007). Longitudinal Tracking of FTLD: Toward Developing Clinical Trial Methodology. Alzheimer Disease \& Associated Disorders, 21(4), S58. https://doi.org/10.1097/WAD.0b013e31815bf69d

López Higes, R., Rubio Valdehita, S., Prados Atienza, J. M., \& Galindo Fuentes, M. (2013). Reserva cognitiva y habilidades lingüísticas en mayores sanos. Rev. neurol. (Ed. impr.), 97-102. https://pesquisa.bvsalud.org/portal/resource/pt/ibc114407

Lozano, A., \& Ostrosky-Solís, F. (2006). Efecto de la edad y la escolaridad en la fluidez verbal semántica: Datos normativos en población hispanohablante. Revista

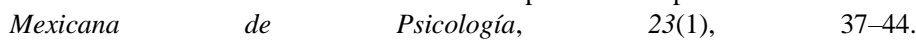
https://www.redalyc.org/pdf/2430/243020646005.pdf

Matías-Guiu, J. A., \& García-Ramos, R. (2013). Afasia progresiva primaria: Del síndrome a la enfermedad. Neurología, 28(6), 366-374. https://doi.org/10.1016/j.nrl.2012.04.003

Ministerio de Desarrollo Social, \& Subsecretaría de Evaluación Social. (2015). Encuesta de Caracterización Socioeconómica Nacional 2015. Adultos Mayores: Sintesis de resultados. Encuesta CASEN. http://observatorio.ministeriodesarrollosocial.gob.cl/storage/docs/casen/2015/CA SEN_2015_Resultados_adultos_mayores.pdf

Morris, J. C. (1997). Clinical Dementia Rating: A Reliable and Valid Diagnostic and Staging Measure for Dementia of the Alzheimer Type. International Psychogeriatrics, 9(S1), 173-176. https://doi.org/10.1017/S1041610297004870

Paolieri, D., Marful, A., Morales, L., \& Bajo, M. T. (2018). The modulating effect of education on semantic interference during healthy aging. PLOS ONE, 13(1), e0191656. https://doi.org/10.1371/journal.pone.0191656

Saá, N. (2006). Lenguaje y envejecimiento. Departamento de Neurología, Pontificia Universidad Católica de Chile. http://publicacionesmedicina.uc.cl/cuadernos/2006/lenguaje.pdf 
Sadowsky, S., \& Martínez-Gamboa, R. (2008). The Modern Spanish Frequency Dictionary-Written Sources (DIFCAM-W).

Sapolsky, D., Bakkour, A., Negreira, A., Nalipinski, P., Weintraub, S., Mesulam, M.-M., Caplan, D., \& Dickerson, B. C. (2010). Cortical neuroanatomic correlates of symptom severity in primary progressive aphasia. Neurology, 75(4), 358-366. https://doi.org/10.1212/WNL.0b013e3181ea15e8

Sapolsky, D, Domoto-Reilly, K., \& Dickerson, B. C. (2014). Use of the Progressive Aphasia Severity Scale (PASS) in monitoring speech and language status in PPA. Aphasiology, 28(8-9), 993-1003. https://doi.org/10.1080/02687038.2014.931563

Savage, S., Hsieh, S., Leslie, F., Foxe, D., Piguet, O., \& Hodges, J. R. (2013). Distinguishing Subtypes in Primary Progressive Aphasia: Application of the Sydney Language Battery. Dementia and Geriatric Cognitive Disorders, 35(3-4), 208-218. https://doi.org/10.1159/000346389
Serrano, C., Martelli, M., Harris, G., Tufró, G., Ranalli, C., Taragano, F., Tamaroff, L., \& Allegri, R. F. (2005). Afasia progresiva primaria: Variabilidad clínica. Análisis de 15 casos. Revista de Neurología, 41(9), 527-533. https://doi.org/10.33588/rn.4109.2005028

Soares, E. C. S., \& Ortiz, K. Z. (2009). Influence of schooling on language abilities of adults without linguistic disorders. Sao Paulo Medical Journal, 127, 134-139. https://doi.org/10.1590/S1516-31802009000300005

Véliz, M., Riffo, B., \& Arancibia, B. (2010). Envejecimiento cognitivo y procesamiento del lenguaje: Cuestiones relevantes. RLA. Revista de lingüística teórica y aplicada, 48(1), 75-103. https://doi.org/10.4067/S071848832010000100005

Zanin, L., Ledezma, C., Galarsi, F., \& De Bortoli, M. Á. (2010). Fluidez verbal en una muestra de 227 sujetos de la región Cuyo (Argentina). 21, 207-219. https://www.redalyc.org/pdf/184/18415426014.pdf 